\title{
Up-gradient transport in a probabilistic transport model
}

\author{
Gavnholt, J.; Juul Rasmussen, J.; Garcia, O.E.; Naulin, V.; Nielsen, A.H.
}

Published in:

Physics of Plasmas

Link to article, DOI:

$10.1063 / 1.2001403$

Publication date:

2005

Document Version

Publisher's PDF, also known as Version of record

Link back to DTU Orbit

Citation (APA):

Gavnholt, J., Juul Rasmussen, J., Garcia, O. E., Naulin, V., \& Nielsen, A. H. (2005). Up-gradient transport in a probabilistic transport model. Physics of Plasmas, 12(8), 084501. https://doi.org/10.1063/1.2001403

\section{General rights}

Copyright and moral rights for the publications made accessible in the public portal are retained by the authors and/or other copyright owners and it is a condition of accessing publications that users recognise and abide by the legal requirements associated with these rights.

- Users may download and print one copy of any publication from the public portal for the purpose of private study or research.

- You may not further distribute the material or use it for any profit-making activity or commercial gain

- You may freely distribute the URL identifying the publication in the public portal

If you believe that this document breaches copyright please contact us providing details, and we will remove access to the work immediately and investigate your claim. 


\title{
Up-gradient transport in a probabilistic transport model
}

\author{
J. Gavnholt, J. Juul Rasmussen, O. E. Garcia, V. Naulin, and A. H. Nielsen \\ Association EURATOM-Ris $\varnothing$ National Laboratory \\ Optics and Plasma Research, OPL-128 Risф \\ DK-4000 Roskilde, Denmark
}

(Dated: June 24, 2005)

\begin{abstract}
The transport of particles or heat against the driving gradient is studied by employing a probabilistic transport model with a characteristic particle step length that depends on the local concentration and heat gradient. When the gradient is larger than a prescribed critical value, the standard deviation of the step size is large compared to its value when the gradient is below critical. For symmetric as well as asymmetric off-axis fuelling, the model is capable of producing profiles peaking at the axis. Additionally, profile consistency is obtained over a broad ranges of source strengths. These results supplement recent works by van Milligen $e t$ al. (Phys. Plasmas 11, 3787 (2004)), which applied Lévy distributed step sizes in the case of super critical gradients to obtain the up-gradient transport.
\end{abstract}


The cross-field transport of particles and heat in magnetically confined hot plasmas is an outstanding issue of high complexity in contemporary fusion plasma research and it is still far from being understood. It is composed of several elements including classical and neo-classical collisional diffusion and anomalous turbulent transport. Several "strange" features characterize the latter including up-gradient transport (transport in the direction of the gradient), profile resilience and consistency (the existence of stiff profiles that are only weakly dependent of the fuelling rate and location), rapid transport phenomena (transient transport events that are significantly faster than the diffusive transport derived from the background gradients). These features cannot consistently be explained by simple "Fickian" diffusion, in which the transport is assumed to be governed by diffusivities.

Recently, van Milligen et al. ${ }^{1,2}$ have proposed a probabilistic model for the description of the transport and the evolution the particle density profile in plasmas with external sources. The model is based on an explicit time and space dependent particle step size probability density function (PDF). This PDF is assumed to depend on the local particle density gradient. When the gradient is below a critical value, the PDF is a Gaussian distribution corresponding to a normal diffusive process that mimics collisional diffusion. However, when the gradient is larger than this critical value, turbulence is assumed to be dominating the transport. Van Milligen et al. argued that the ensuing anomalous transport is dominated by a "super-diffusive" behavior, where the step length in the process is not distributed according to a standard Gaussian distribution, but according to a Lévy distribution. This distribution is characterized by a broad, algebraically decaying tail for large events, implying that there is no characteristic length scale. It signals that long-range correlations are inherent in the turbulent transport and implies that the confinement time will be proportional to some power of the system size, which is not necessarily quadratic. Van Milligen et al. ${ }^{2}$ have solved this model for different situations with various source distributions and have found that it reproduces several of the "strange" features mentioned above. It was strongly emphasized that the Lévy type particle step PDF is essential for the observed characteristics.

The model proposed by van Milligen and co-workers ${ }^{1,2}$ can be expressed in terms of a general master equation, here governing the evolution of the particle density profile, $n(x, t)$ :

$$
\frac{\partial n(x, t)}{\partial t}=S(x)-\frac{n(x, t)}{\tau_{\mathrm{D}}}+\int_{0}^{1} P\left(x-x^{\prime}, x^{\prime} ; t\right) \frac{n\left(x^{\prime}, t\right)}{\tau_{\mathrm{D}}} d x^{\prime},
$$

where $P\left(x-x^{\prime}, x^{\prime} ; t\right)$ is the particle step probability density function, $S(x)$ designates the source of particles, $\tau_{\mathrm{D}}$ is a typical diffusion time scale, and will be taken as $\tau_{\mathrm{D}}=1$ (cf. Refs. 1,2). If 
$P\left(x-x^{\prime}, x^{\prime}\right)$ is a Gaussian with a standard deviation $\sigma$ much smaller than the size of the domain in which it is considered, the master equation (1) is reduced to a standard diffusion equation in the interior of the domain by using a Taylor expansion of the particle density around $x^{\prime}=x$ (cf. Ref. 1):

$$
\frac{\partial n(x, t)}{\partial t}=\frac{\sigma^{2}}{\tau_{\mathrm{D}}} \frac{\partial^{2} n(x, t)}{\partial x^{2}}+S(x)
$$

with the diffusion coefficient $D=\sigma^{2} / \tau_{\mathrm{D}}$.

To invoke the critical gradient effect, van Milligen et al. chose $P$ as composed of two different distributions depending on whether the gradient is below or above the critical one, i.e.,

$$
P\left(x-x^{\prime}, x^{\prime} ; t\right)=\xi\left(x^{\prime}, t\right) P_{1}\left(x, x^{\prime}\right)+\left(1-\xi\left(x^{\prime}, t\right)\right) P_{2}\left(x, x^{\prime}\right)
$$

where

$$
\xi\left(x^{\prime}, t\right)=\Theta\left(\left|\frac{d n\left(x^{\prime}, t\right)}{d x}\right|-\kappa_{\text {crit }}\right) .
$$

$\Theta(x)$ is the standard Heaviside step function and $\xi\left(x^{\prime}, t\right)$ will be either one or zero depending on the local value of the gradient with respect to a prescribed critical value $\kappa_{\text {crit }}$ above which the diffusion is anomalous. Van Milligen et al. employed a Gaussian distribution for $P_{2}$ and a Cauchy distribution for $P_{1}$, viz.

$$
P_{1}\left(x, x^{\prime}\right)=\frac{\sigma_{1}}{\pi\left[\sigma_{1}^{2}+\left(x-x^{\prime}\right)^{2}\right]}, \quad P_{2}\left(x, x^{\prime}\right)=\frac{1}{2 \sigma_{2} \sqrt{\pi}} \exp \left[\frac{-\left(x-x^{\prime}\right)^{2}}{4 \sigma_{2}^{2}}\right] .
$$

We have examined the model of van Milligen et al. in order to investigate the sensitivity of the results on the assumed particle step PDFs. In particular, we have considered the model where both $P_{1}$ and $P_{2}$ are Gaussians, but with different standard deviations, $\sigma_{1}>\sigma_{2}$. For this case the master equation (1) may be reduced to the following diffusion equation [cf. Eq. (2)]

$$
\frac{\partial n(x, t)}{\partial t}=\frac{1}{\tau_{\mathrm{D}}}\left\{[1-\xi(x, t)] \sigma_{2}^{2}+\xi(x, t) \sigma_{1}^{2}\right\} \frac{\partial^{2} n(x, t)}{\partial x^{2}}+S(x),
$$

where $\xi(x, t)$ is defined in Eq. (4). Note that this equation resembles the critical gradient diffusion model introduced by Imbeaux et al. ${ }^{3}$ that is often used in modelling the transport of heat as well as particle density in magnetically confined plasmas (see e.g., Refs. 4,5 and works cited therein). In these works it is argued that the diffusion coefficient is classical below a certain critical gradient and an anomalous or turbulent component is added when the gradient surpasses the critical value. The diffusivity of the anomalous component is assumed to increase with the departure of the gradient from the critical one, thus providing a strong anomalous contribution to the diffusive transport. 
Indeed, there are ample experimental evidence for the existence of such a critical gradient, see, e.g., Refs. 6,7.

We have solved the master equation 1 on the bounded domain $0 \leq x \leq 1$ with absorbing boundaries, i.e., a stationary profile may be achieved when the total particle flux through the boundaries equals the integrated source rate. Here we will concentrate on the cases with off-axis sources. First we consider two sources placed symmetrically with respect to the axis:

$$
S(x)=\frac{S_{0}}{2}\left[\frac{1}{\sqrt{2 \pi} w} \exp \left(-\frac{\left(x-x_{1}\right)^{2}}{2 w^{2}}\right)+\frac{1}{\sqrt{2 \pi} w} \exp \left(-\frac{\left(x-x_{2}\right)^{2}}{2 w^{2}}\right)\right] .
$$

We have used the parameters:

$$
\sigma_{2}=0.02, \kappa_{\text {crit }}=50, S_{0}=0.2, w=0.025, x_{1}=0.3, x_{2}=0.7
$$

while $\sigma_{1}$ is varied. In Fig. 1 we show the particle density profile in the stationary state for different particle step PDFs. In all cases the particle density profile is clearly peaked in the center signifying up-gradient transport. However, for two Gaussian PDFs with $\sigma_{1}=2 \sigma_{2}$ the profile is steeper than the corresponding profile obtained with the Cauchy-Gauss distributions in Eq. (5) shown for comparison. By increasing the value of $\sigma_{1}$ we obtain a profile almost indistinguishable from the one obtained by the Cauchy-Gauss distributions. We thus observe that the Lévy type distribution is not necessary for providing up-gradient transport. The crucial ingredient is simply the existence of two different step sizes in the transport peocess. Consistently, we do not observe peaking for two distributions with the same standard deviation, as was also pointed out in Ref. 2.

For the case of two Gaussian distributions we can estimate the gradient of the profile by using the diffusion equation (6) and balancing the flux in the anomalous channel with the source term:

$$
\int_{0}^{1} D_{1} \frac{d^{2} n(x, t)}{d x^{2}} d x=-\int_{0}^{1} S(x) d x
$$

implying

$$
2 D_{1}\left|\frac{d n(x, t)}{d x}\right|_{x=0,1}=S_{0},
$$

where $D_{1}=\sigma_{1}^{2} / \tau_{\mathrm{D}}$. This results in $|d n / d x|=62.5$ at the boundaries for the case in Fig. 1 with $\sigma_{1}=2 \sigma_{2}$, agreeing with the observed value and significantly larger than the prescribed critical gradient $\kappa_{\text {crit }}=50$. From Eq. (8) we observe that the steady state gradient will decrease for a larger step size standard deviation $\left(\sigma_{1}\right)$ or a smaller source. By decreasing the source we have indeed observed a decrease in the gradient. However, for a sufficiently small value of $S_{0}$ the up-gradient 
transport disappears. For a fixed fuelling rate we deduce from the preceding argumentation that a consistent particle density profile may be obtained when the transport in the anomalous channel at the critical particle density gradient exceeds the integrated source rate $S_{0}$, i.e.,

$$
2 D_{1} \kappa_{\text {crit }} \geq S_{0}
$$

The concentration gradient will then be close to $\kappa_{\text {crit }}$. This is in agreement with the observation in Fig. 1 for $\sigma_{1}=4 \sigma_{2}$. When the requirement (9) is satisfied we may expect stiff profiles for varying source rates, which will be demonstrated below. We should mention that these arguments agree with the results from the so-called turbulent equipartition principle. ${ }^{8,9}$

We have also considered the case of one asymmetric particle source:

$$
S(x)=S_{0} \frac{1}{\sqrt{2 \pi} w} \exp \left[-\frac{\left(x-x_{1}\right)^{2}}{2 w^{2}}\right]
$$

with $S_{0}=0.2, w=0.05$. The results for two different values of $\sigma_{1}$ are presented in Fig. 2. Again we observe up-gradient transport and particle density peaking. The detailed structure clearly depends on the parameters, but only weakly on the source position, as long as it is well inside the box. For $\sigma_{1}=2 \sigma_{2}$ the profile is asymmetric, but by decreasing $S_{0}$ or increasing $\sigma_{1}$ to comply with Eq. (9) we obtain a symmetric profile with the gradient close to $\kappa_{\text {crit }}$.

The dynamical evolution of the profile when the off-axis symmetric sources are turned on at $t=0$ is shown in Fig. 3. We observe that that significant up-gradient transport sets in when the steepest gradients have reached the critical value, $\kappa_{\text {crit. }}$. The profile then rapidly approaches the peaked equilibrium profile. We further investigated the response of the steady state particle density profile when it is perturbed by removing the particles for $x<0.25$ instantaneously at $\tau=0$. The subsequent evolution is shown in Fig. 4. We observe a very fast transmission of the perturbation to the center of the profile and the peak is slightly shifted to higher $x$ values. This behavior is in close agreement with the similar investigation by van Milligen et al. ${ }^{1}$ using a Cauchy step size distribution for the anomalous transport channel.

A characteristic experimental observation is the stiffness of the profile. This means that the profile is only very weakly dependent on the source strength. In Fig. 5 we demonstrate the profile consistency by plotting the central particle density, $n(x=0.5)$, for different source rates, $S_{0}$, and various particle step size distributions for the anomalous channel. It is observed that the peak particle density is roughly constant over a broad range of source rates $S_{0}$, for the case of GaussianCauchy distributions as well as for two Gaussians when $\sigma_{1}>2 \sigma_{2}$. This is a signature of profile 
consistency. The gradient is close to the critical gradient in accordance with the discussion in connection with Fig. 1. Note that we obtained a similar profile stiffness when using an asymmetric off-axis fuelling as in Fig. 2.

In conclusion, we have demonstrated that the essential feature for obtaining profile peaking and consistency in transport models is the existence of a step size PDF regulated by a critical gradient and not the detailed functional shape of this PDF. Above the critical gradient, where the anomalous transport channel operates, the effective step size must be substantially larger than the step size of the classical transport channel effective below the critical gradient, where collisional transport dominates. It is not necessary to have a Lévy type PDF for the anomalous transport channel as emphasized in a recent work by van Milligen et al. ${ }^{1,2}$ It should be emphasized, however, that the approach taken by van Milligen et al. indeed includes features not captured by Gaussian statistics, as, e.g., confinement time scalings, and our results should thus be regarded as a supplement to their work. Additionally, the formulation of the problem as a probabilistic model described by a general master equation contains a much broader range of applicability than the diffusion equation approach. It is obvious that the probabilistic model opens up for treating transport processes that are governed by generalized step size distributions and additionally also allows for the investigations of non-Markovian memory effects. This approach may prove valuable for the development of new transport codes providing better understanding of transport in magnetized plasmas and the observed "strange" features of profile evolution.

This work was supported by the Danish Center for Scientific Computing through grants no. CPU-1101-08 and CPU-1002-17. O. E. Garcia has been supported by financial subvention from the Research Council of Norway. 
1 B. Ph. van Milligen, R. Sánchez, and B. A. Carreras, Phys. Plasmas 11, 2272 (2004).

2 B. Ph. van Milligen, B. A. Carreras, and R. Sánchez, Phys. Plasmas 11, 3787 (2004).

3 F. Imbeaux, F. Ryter and X. Garbet, Plasma Phys. Contr. Fusion 43, 1503 (2001).

4 F. Ryter, C. Angioni, M. Beurskens et al., Plasma Phys. Contr. Fusion 43, A323 (2001).

5 A. G. Peeters, C. Angioni, M. Apostoliceanu, F. Jenko, F. Ryter and the ASDEXUpgrade Team, Phys. Plasmas 12, 022505 (2005).

${ }^{6}$ C. T. Hoang, C. Bourdelle, X. Garbet, G. Giruzzi, T. Aniel, M. Ottaviani, W. Horton, P. Zhu, and R. V. Budny, Phys. Rev. Lett. 87, 125001 (2001).

7 D. R. Baker, C. M. Greenfield, K. H. Burrell et al., Phys. Plasmas 8, 4128 (2001).

8 V.V. Yankov and J. Nycander, Phys. Plasmas 4, 2907 (1997).

9 V. Naulin, J. Nycander and J. Juul Rasmussen, Phys. Rev. Lett. 81, (1998). 
FIG. 1: (Color online) Solutions of the master equation (1) for the particle density profile with off-axis fuelling for different step size distributions. Dashed line (black): Two Gaussian distributions with the step sizes: $\sigma_{1}=0.04$ and $\sigma_{2}=0.02$. Solid line (blue): Two Gaussian distributions with $\sigma_{1}=0.08$ and $\sigma_{2}=0.02$. Dashed-dotted line (red): A Cauchy distribution with $\sigma_{1}=0.04$ and a Gaussian with $\sigma_{2}=0.02$ (as used in Ref. 2, Fig 3). The two sources are symmetrically placed and indicated by the thin black line.

FIG. 2: (Color online) The particle density profile for asymmetric forcing with the source, shown by the thin black curve, placed at the left side. The parameters are the same as in Fig. 1, dashed line (black): $\sigma_{1}=2 \sigma_{2}$, and solid line (blue): $\sigma_{1}=4 \sigma_{2}$.

FIG. 3: (Color online) Dynamical evolution of the particle density profile when the two sources, placed at $x=0.3$ and $x=0.7$, are introduced at $t=0$. The Gaussian step size distributions have $\sigma_{1}=2 \sigma_{2}=0.04$, and $S_{0}=0.2$.

FIG. 4: (Color online) Response of the particle density profile to a "cooling" perturbation applied on the left hand side to the steady state profile at $\tau=0$. Contours of constant particle density are plotted in the $x-t$ plane with contour spacing $=1.5$. The two symmetric off-axis sources are placed at $x=0.3$ and $x=0.7$. The step size Gaussian distributions have $\sigma_{1}=2 \sigma_{2}=0.04$, and $S_{0}=0.2$.

FIG. 5: (Color online) Demonstration of profile "stiffness", shown by the dependence of the central particle density $n(x=0.5)$ on the source strength $S_{0}$, for the cases where the anomalous transport channel is characterized by a Cauchy distribution and the cases where it is characterized by a Gaussian with the values of $\sigma_{1}$ as shown in the legend and $\sigma_{2}=0.02$ 


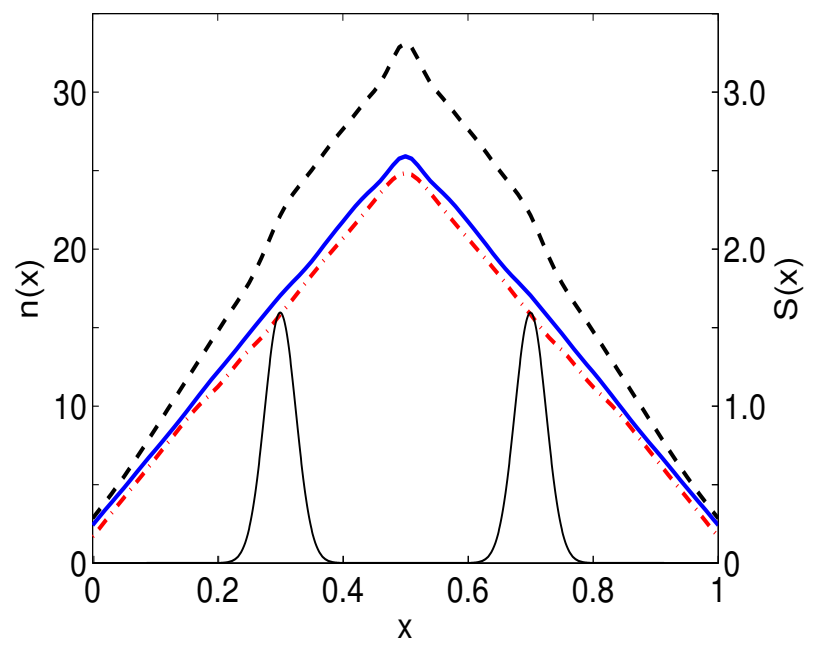

FIGURE 1.

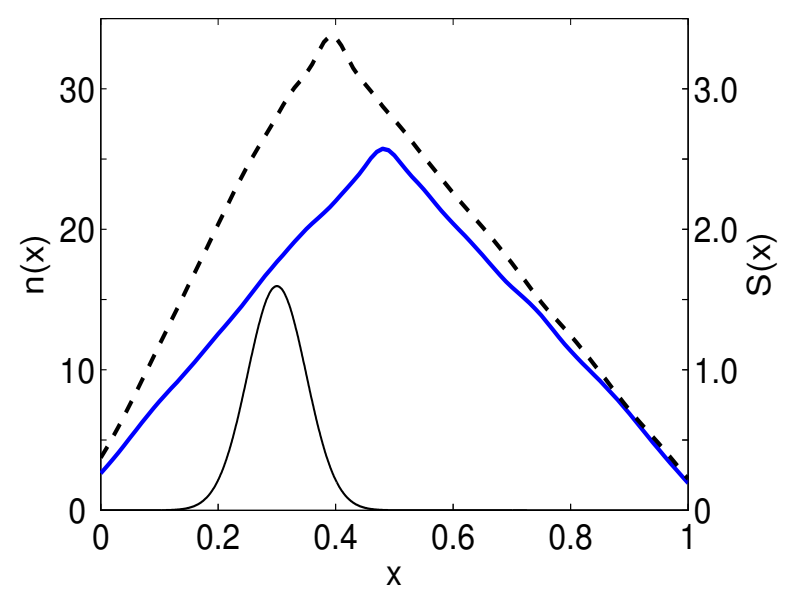

FIGURE 2. 


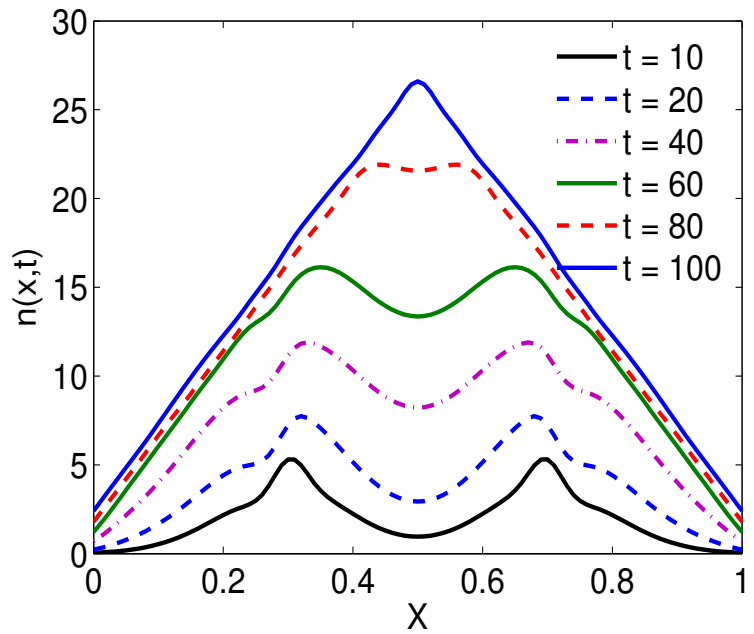

FIGURE 3.

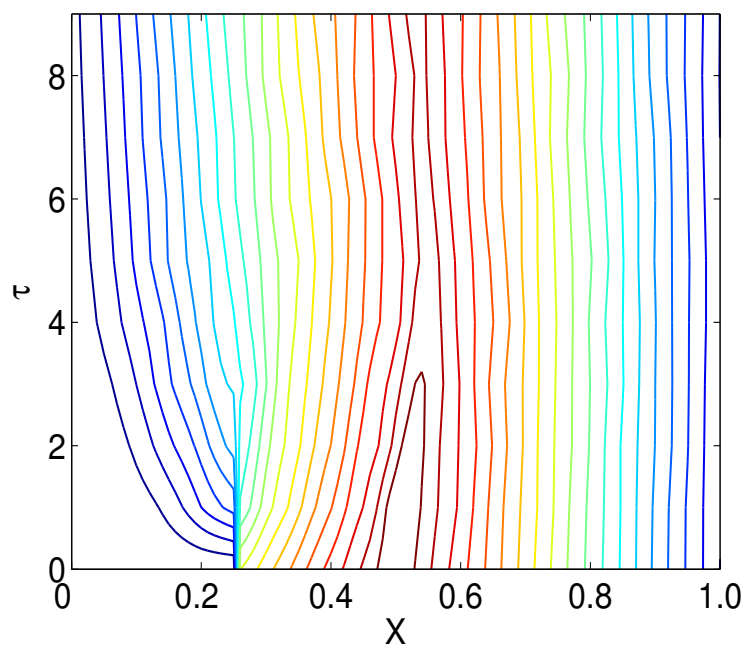

FIGURE 4. 


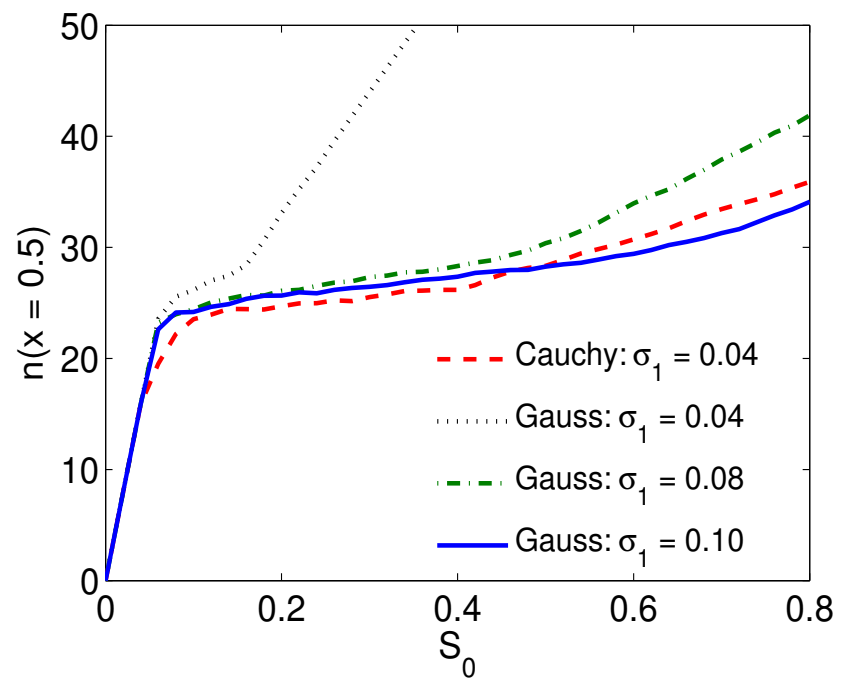

FIGURE 5. 